\title{
Coming across a novel copper oxide 2D framework during the oxidation of $\mathrm{Cu}(111)$
}

\author{
Sonia Matencio, ${ }^{\dagger}$ Esther Barrena and Carmen Ocal ${ }^{*}$ \\ Institut de Ciència de Materials de Barcelona (ICMAB-CSIC). Campus de la UAB, 08193 Bellaterra, Spain.
}

*Corresponding author E-mail: cocal@icmab.es

† Present Address: Institute of Experimental and Applied Physics, University of Regensburg, 93053 Regensburg, Germany.

\begin{abstract}
Two dimensional copper oxides obtained on $\mathrm{Cu}(111)$ by air-enriched argon sputtering plus annealing have been measured at room temperature by means of scanning tunneling microscopy (STM) and atomic force microscopy (AFM) under ultra-high vacuum (UHV) conditions. Depending on the oxygen content different oxide frameworks and diverse stoichiometric metal/oxide interfaces exist. In particular, we report on a novel open honeycomb structure with a large unit cell which is modeled as a two dimensional network made out of $\mathrm{Cu}_{3} \mathrm{O}$ units. This lattice coexists with other oxide structures richer in oxygen and is suggested to develop towards these denser phases by oxygen incorporation.
\end{abstract}

\section{Introduction}

The electrical, mechanical, thermal and chemical properties of many technological devices are often intimately dependent on the structure, composition and morphology of internal metal/oxide interfaces. Mastering interface fabrication with nanometer precision will lead to progress in numerous fields where nanostructured interfaces in the form of controlled networks or ultrathin films will play an active role in novel integrated platforms. On the other hand, advances in nanotechnology depend on the development of innovative and efficient procedures for device fabrication down to the nanometer scale. The ability of tailoring interfaces without the need of additional building blocks, i.e., from adequate handling of the surfaces themselves is of particular interest. In this sense, though important advances have taken place for local oxidation using scanning probe-based techniques, ${ }^{1,2}$ the scalability and manufacturing speed are far from being practical for applications. An attractive route for the simple creation of two dimensional metal/oxide architectures is the use of standard surface treatments (ion milling, gas exposure, thermal treatment...) for in-situ creating two dimensional networks consisting of one atom thick inorganic surface compounds. Adjusting the preparation parameters to change the layer stoichiometry would permit controlling the pattern compactness from packed layers to porous frameworks.

The chemical reaction of metals with air is of unpayable relevance and especially important in catalysis, corrosion and corrosion protection. In the case of copper, one of the most common coin metals used in the industry, the interaction of the metal surface with gaseous oxygen plays a detrimental role in other applications, as in microelectronics, where it is an active element. Nevertheless, because of their electrical characteristics, copper oxide ultrathin films are good candidates for uses as low resistance electrodes, catalysts, sensing materials and semiconductor materials for solar cell transformation. ${ }^{3-8}$ They predominantly exist in two forms, $\mathrm{Cu}_{2} \mathrm{O}$ and $\mathrm{CuO}$, both intrinsic p-type semiconductors that can be synthesized from the oxidation of $\mathrm{Cu}$ metal at low processing costs. In particular, $\mathrm{Cu}_{2} \mathrm{O}$ has been shown to be an effective photo-catalyst for water splitting to $\mathrm{H}_{2}$ and $\mathrm{O}_{2}$ under visible light ${ }^{9}$ and, due to a suitable direct band gap, $\mathrm{Eg} \approx 2.14 \mathrm{eV}$, susceptible of being modified by nano-structuration, it is also considered functional material for photovoltaic and photo-electrochemical device applications. ${ }^{10}$ However, it has been argued that device efficiencies are limited in part because there is no method for fabricating a reproducible, stoichiometric interface between the copper oxide and other heterojunction partners. ${ }^{11}$

With the aim of increasing knowledge of well-defined and stoichiometric metal/oxide interfaces, we investigate here diverse two dimensional cooper oxides obtained by air-enriched argon sputtering plus annealing of $\mathrm{Cu}$, an efficient and scalable methodology. Laterally heterogeneous surfaces consisting of one layer thick copper oxide regions coexisting with bare Cu areas have been investigated at room temperature (RT) by means of STM and AFM in the frequency modulation mode (FM-AFM) under UHV conditions. The combination of these local probe techniques permits elucidating the interface structure at atomic level and disentangle electronic from topographic information.

In addition to some already described cooper oxide structures of varying stoichiometry, ${ }^{12-16}$ we report here on a novel open honeycomb structure with a large unit cell (lattice parameter $\approx 1.3 \mathrm{~nm}$ ). We propose a model in which this low density oxide is made out of $\mathrm{Cu}_{3} \mathrm{O}$ and is suggested to develop to denser phases by oxygen incorporation.

\section{Experimental details}

The combined STM/FM-AFM measurements were carried out at RT and base pressure $\sim 5 \times 10^{-10}$ mbar using a commercial Aarhus SPM 150 equipped with KolibriSensor ${ }^{\mathrm{TM}}$ and Nanonis Control System (SPECS Zurich GmbH). The sharp metallic tip (resonance frequency $\mathrm{f}_{0} \approx 1 \mathrm{MHz}$ ) was in-situ cleaned via $\mathrm{Ar}^{+}$sputtering. In the STM images, the tunneling bias voltage is applied to the sample and the tunneling current is collected by the tip, which is grounded. Topographic STM corresponds to constant current imaging. In FM-AFM ${ }^{17}$ the sensor oscillation amplitude was set to $A=200 \mathrm{pm}$ during operation and the frequency shift $\left(\Delta \mathrm{f}=\mathrm{f}-\mathrm{f}_{0}\right)$ was the input signal in a feedback loop for topographic measurements. 
The $\mathrm{Cu}(111)$ single crystal (Matek GmbH, Germany) was prepared by repeated cycles of $\mathrm{Ar}^{+}$sputtering $(1.2 \mathrm{keV}, 9 \mu \mathrm{A})$ plus annealing at $240^{\circ} \mathrm{C}$ during $25-30 \mathrm{~min}$ in a dedicated sample holder located in the scanning probe chamber. Cleanness and ordering of the surface was checked by STM and LEED (a-c of Figure SI1 in ESI). In-plane substrate distances in high resolution images and step heights in large scanned images were employed for in-situ calibration of the piezo scanner. The LEED pattern confirmed a well-ordered and clean surface and was used to stablish the crystal azimuth orientation for the structural analysis of the STM and FM-AFM images. To check that no $\mathrm{O}$ or $\mathrm{C}$ traces remained on the $\mathrm{Cu}(111)$ surface after the employed cleaning process, the same procedure was repeated in a different chamber equipped with XPS (d-f of Figure SI1 in ESI). Partial oxidation of the surface was obtained by air-enriched $\mathrm{Ar}^{+}$bombardment and annealing with same parameters than for substrate cleaning. The pressure in the UHV chamber during oxidation was kept in the $10^{-5}$ mbar range and the introduced mixture of ambient air and Ar (see Figure SI2 in ESI for gas composition) was ionized with the ion gun. Immediately after, the base pressure of $\sim 5 \times 10^{-10}$ mbar was recovered and the sample transferred to the scanning probe microscope.

\section{Results and Discussion}

It is generally agreed that oxygen molecules dissociate at the $\mathrm{Cu}(111)$ surface at room temperature leading to a disordered surface layer. Though complex structures observed at elevated temperature or higher pressures eventually lead to more or less stoichiometric $\mathrm{Cu}_{2} \mathrm{O}$ films, a certainly complicated scenario exists at the initial stages of oxygen incorporation. The reason seems to have it basis on the relationship between copper and copper oxide structural parameters. The bulk-terminated $\mathrm{Cu}_{2} \mathrm{O}(111)$ can be viewed as a stack of trilayers, each consisting of alternated composition (i.e., Cu-O-Cu). ${ }^{18}$ The in-plane lattice is a honeycomb structure with lattice parameter $\mathrm{a}_{\mathrm{Cu} 2 \mathrm{O}(111)} \approx 6 \AA$ where the $\mathrm{Cu}-\mathrm{Cu}$ distance is half of this value $(\approx 3 \AA)$. This distance is considerably larger/smaller (by $15 \%$ and $47 \%$, respectively) than the NN/NNN distance in $\mathrm{Cu}(111), \mathrm{a}_{\mathrm{Cu}(111)}=2.56 \AA$ and $\mathrm{a}_{\mathrm{Cu}(111)} \cdot \sqrt{3}=4.43 \AA$. However, commensurate strained/compressed lattices of such an oxide overlayer are likely to exist. This is the case for reported coincidence lattices $^{19,20}$ where four times the $\mathrm{NNN}$ distance of $\mathrm{Cu}(111)$ coincides with three times the lattice parameter of $\mathrm{Cu}_{2} \mathrm{O}(111)$ (i.e. $\left.4 \mathrm{a}_{\mathrm{Cu}(111)} \cdot \sqrt{ } 3\right) / 3=6 \AA$ ), and seven times the NN distance of the Cu substrate ( $7 \times 2.56 \AA=17.92 \AA$ ) is close to three times the oxide lattice parameter (3 x $5.95 \AA=17.85 \AA$ ).

Different methods have been employed for the oxidation of $\mathrm{Cu}(111)$, the exposure of the clean surface to $\mathrm{O}_{2}$ pressure in UHV plus annealing being the most common one to obtain monolayer oxides. However, small differences in pressure and temperature give to the presence of diverse surface structures. Interestingly, most of these structures were also achieved by reduction of the oxide monolayer or using reactive air injection, ${ }^{12,16}$ indicating their relative stability independently of the preparation methodology. It is however likely that modifying the energetics and kinetics of the oxidation process may lead to new metastable oxide structures. With the aim of pursuing this objective, instead of complete oxidized surfaces, partial coverages of oxide layers on the Cu(111) have been prepared. Our strategy consists in submitting the clean $\mathrm{Cu}(111)$ surface to an air-enriched $\mathrm{Ar}^{+}$sputtering followed by in-situ annealing ( $25 \mathrm{~min}$ at $240^{\circ} \mathrm{C}$ ) and cooling down to RT while removing the oxidizing gas. The low oxygen content of the sputtering atmosphere (see Figure SI2) and interruption of the oxidation process leads to final surfaces laterally heterogeneous formed by oxide patches and clean copper regions.

With this procedure, up to six different phases have been seen to coexist on the same sample surface depending on the local oxygen content: oxygen adsorbed on bare copper, ${ }^{15}$ four structures described so far in the literature, ${ }^{12-16}$ and a new largely open honeycomb (OHC) lattice with a lower oxygen content than the reported phases. ${ }^{21}$ This novel structure, which can be viewed as a $2 \mathrm{D}$ framework, is completely described thanks to its coexistence with the bare $\mathrm{Cu}(111)$, the known phases and the accepted models.
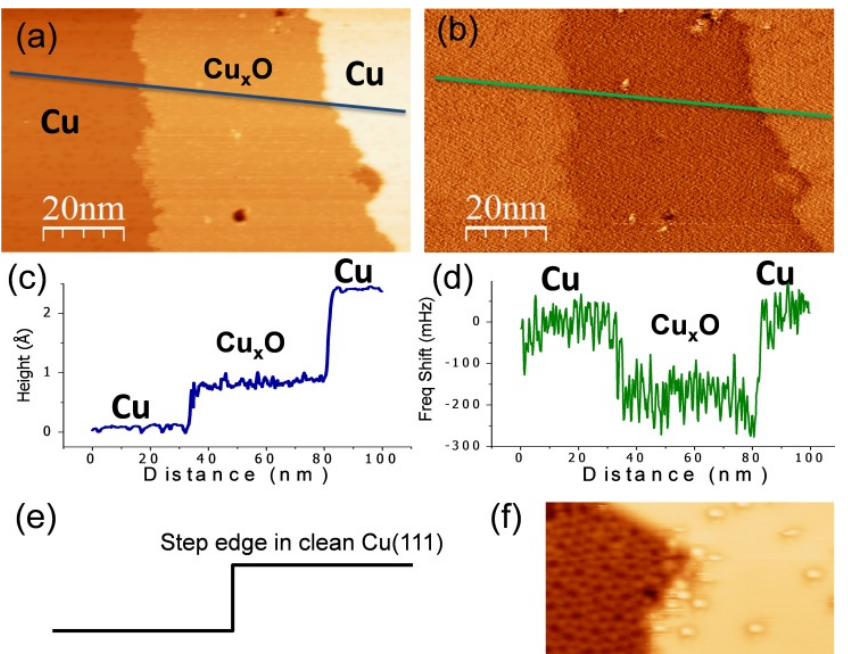

Step region after air/ $\mathrm{Ar}^{+}$oxidation
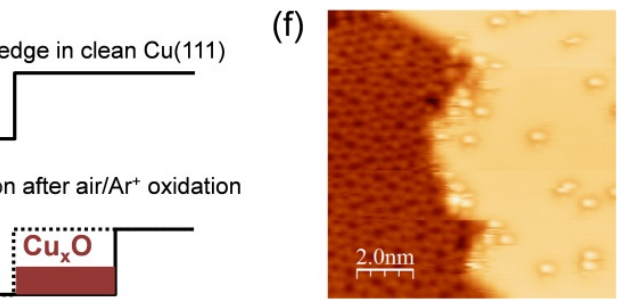

Figure 1. (a) Topographic STM image of partially oxidized Cu(111), (b) simultaneous frequency shift channel and (c)-(d) corresponding line profiles. (e) Schematics of the oxidation process at the step edges. (f) Magnified topographic image showing short range order at the oxide region. STM parameters: (a) and (b) $\mathrm{I}=195 \mathrm{pA}$, Bias $=-1.45 \mathrm{~V}$, (f) $\mathrm{I}=265 \mathrm{pA}$, Bias $=-0.44 \mathrm{~V}$. 
Figure 1 shows the coexistence of copper terraces, covered by some oxygen adsorbed species (see Figure SI3 in ESI), with partial oxidation of the surface. This observation agrees with the nucleation and growth at RT of the oxide layer on the Cu(111) surface, which proceeds by formation of patches at the step edges due to reaction of the impinging oxygen with the copper atoms at the upper terrace (Figure 1e). The oxide patches propagate as oxygen is incorporated to the surface. ${ }^{12,13,14}$ These regions present a large corrugation (0.3-0.8 $\AA$ ) and local order in the form of a nearly hexagonal lattice of $\approx 6 \AA$ (left region of Figure 1f) in average, close to the lattice parameter of $\mathrm{Cu}_{2} \mathrm{O}(111)$ and more than twice that of $\mathrm{Cu}(111)$. The short order of this defective honeycomb lattice is consequence of heterogeneous nucleation starting at different locations. Noticeably, this structure has been observed both during surface oxidation ${ }^{14,15}$ or reduction, ${ }^{12,13}$ pointing to a non-stoichiometric intermediate phase.

In Figure 1, the oxide region is about $\sim 1 \AA$ lower than that of the Cu(111) upper terrace, but depending on the STM measuring conditions it can be observed even below the level of the lower terrace. ${ }^{14,16}$ This height would correspond to a thickness much lower than steps or inter-plane distances for $\mathrm{Cu}(111)$ or $\mathrm{Cu}_{2} \mathrm{O}(111), \mathrm{d}_{\mathrm{Cu}(111)}=2.1 \AA$ and $\mathrm{d}_{\mathrm{Cu} 2 \mathrm{O}(111)} \approx 3 \AA$, ${ }^{22}$ respectively. However, it is known that topographic STM measurements can give erroneous relative heights for heterogeneous surfaces presenting different local density of states (LDOS). ${ }^{23}$ This is particularly pertinent here, as oxide surfaces have a LDOS where electronic states are generally more localized than on the pure metal ${ }^{24,25}$ and have an associated gap. As it can be seen in Figure $1 \mathrm{~b}-1 \mathrm{~d}$, a different frequency shift is also measured between bare and oxidized copper regions. The tip-surface interaction is weak at Cu while attractive and stronger $(\approx-200 \mathrm{mHz})$ at the oxide. This behavior likely reflects the decrease in tip-sample distance taking place to keep constant the tunneling current when passing from the copper surface to the metal-oxide layer.
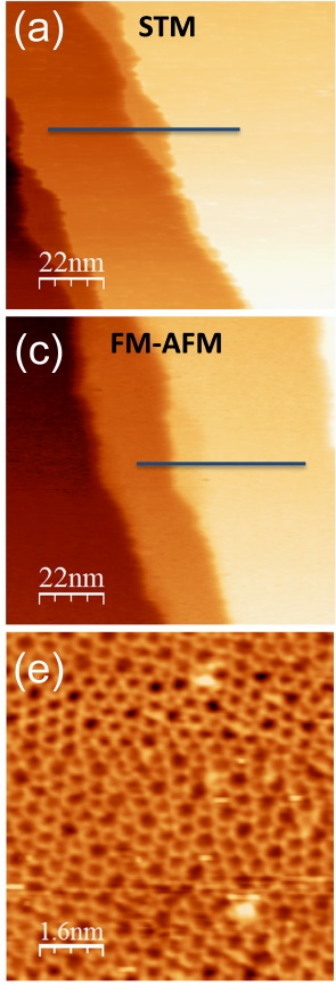

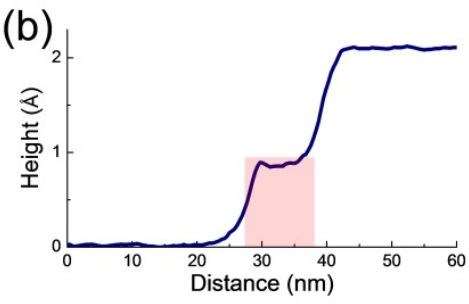

(d)
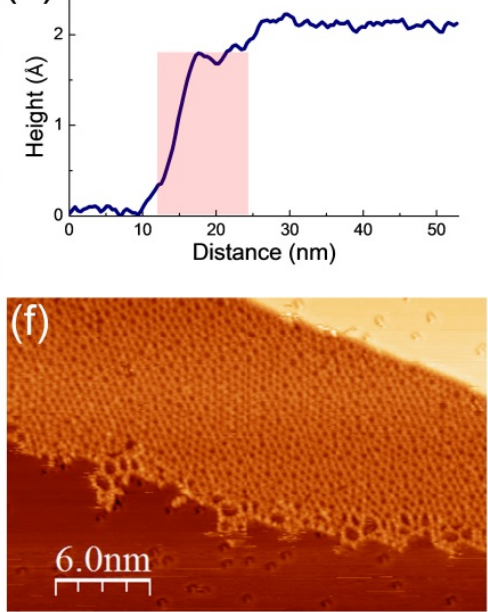

Figure 2. Topographic STM (a) and FM-AFM (c) images containing a small region of copper oxide at the step region. The corresponding line profiles are shown in (b) and (d), with oxide regions highlighted in pink. (e), (f) Short order honeycomb structure. STM parameters: (a) I = 420 pA, Bias $=+1.08 \mathrm{~V}$, (c) $\Delta \mathrm{f}=-1.00 \mathrm{~Hz}$, Bias $=+0.08 \mathrm{~V}, \mathrm{~A}=200 \mathrm{pm}$, (e) $\mathrm{I}=194 \mathrm{pA}$, Bias $=-0.47 \mathrm{~V}$, (f) $\mathrm{I}=100 \mathrm{pA}$, Bias $=+0.74 \mathrm{~V}$.

In order to get some insight on the true topographic relief we compare the apparent height obtained by constant current STM and the topography as measured by FM-AFM, which is considered to provide more accurate height values. ${ }^{26}$ Figures $2 \mathrm{a}$ and $2 \mathrm{c}$ show images obtained by both methods as well as the corresponding line profiles ( $2 \mathrm{~b}$ and $2 \mathrm{~d}$ ) selected to cross the oxide patch between neighbor copper terraces. The oxide thickness is $\approx 1.75 \AA$ in FM-AFM, much reliable that the apparent value of $\approx 0.9 \AA$ in constant current STM. The difference in height of $\approx 0.3 \AA$ between the copper terrace and the short order honeycomb or defective layer in FM-AFM is compatible with its open mesh and large corrugation (Figure 2e). As already reported, ${ }^{13}$ it contains intermixed pentagons and heptagons (5-7 defects) within the hexagonal lattice (see also Figure 5b and full description of Figure SI4 in ESI). These topological defects have been proposed to be analogous to the Stone-Wales (S-W) defects observed in graphene ${ }^{27,28}$ and reflect a non-stoichiometric phase. Interestingly, larger defects in the form of lace edging shapes forming a fretwork-like border are accumulated at the boundary of the oxide patch (Figure 2f). This fact is associated to the lowest oxygen content phase debated at the last part of the present discussion. 


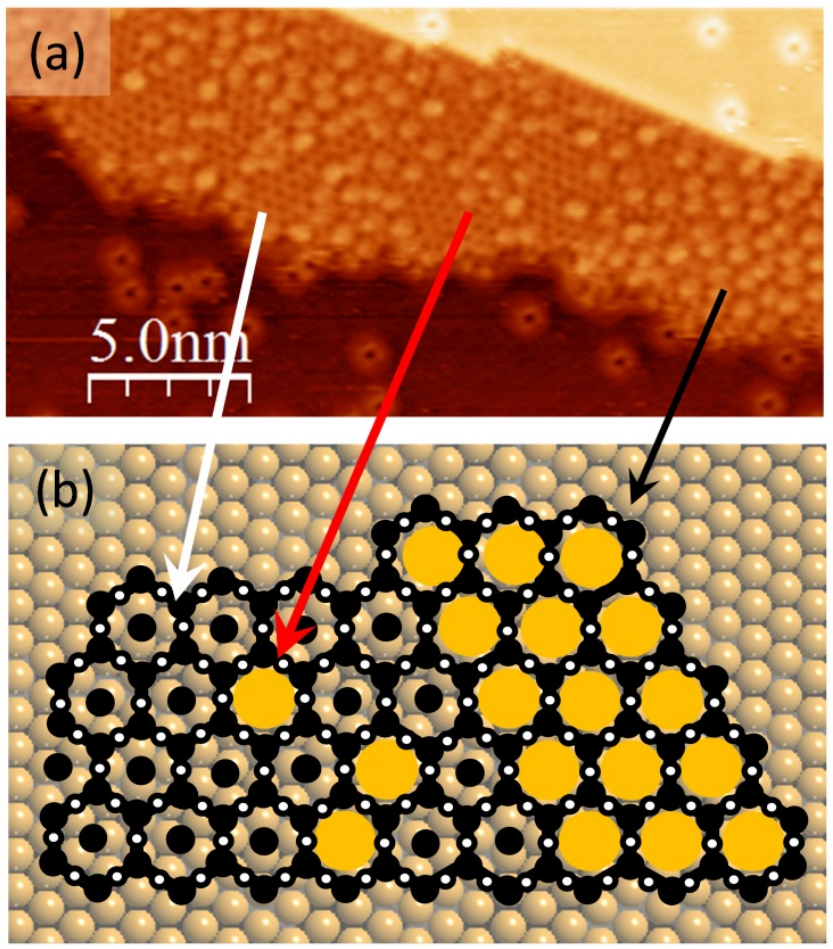

Figure 3. Topographic STM image (a) of the O-deficient oxide structure. (b) Schematics of different stages in (a). Atoms in the oxide layer: oxygen (black), copper (white) and oxygen vacancies (orange). Substrate copper atoms are represented by golden balls. STM parameters: $\mathrm{I}=120 \mathrm{pA}$, Bias $=+$ $1.51 \mathrm{~V}$.

Figure 3 shows a surface region containing a different oxide structure, the so called O-deficient honeycomb. This structure has been described as consisting of a well-developed honeycomb lattice in which oxygen vacancies at the center of the hexagons produce an increase in the electronic density at the surrounding $\mathrm{Cu}$ atoms. ${ }^{12}$ This charge unbalance is evidenced in STM images as bright protrusions (red arrow). Grouped neighboring oxygen vacancies form well-defined hexagonal areas that can be seen (black arrow) coexisting with defect-free or oxygen centered honeycomb regions (white arrow) with same lattice parameter $\approx 6 \AA$ than the $\mathrm{Cu}_{2} \mathrm{O}(111)$ plane (see also Figure SI5). As illustrated in Figure 3b, accommodating different amount of oxygen, the O-deficient overlayer evolves to the full and compact $\mathrm{Cu}_{2} \mathrm{O}(111)$-like strained layers denoted as " 29 " and " 44 " structures. ${ }^{20}$ These two structures are identified in Wood's notation as ( $\sqrt{13} \mathrm{R} 46.1^{\circ} \times 7 \mathrm{R} 21.8^{\circ}$, " $\left.29^{\prime \prime}\right)$ and ( $\sqrt{77} \mathrm{R} 5.8^{\circ} \times \sqrt{21} \mathrm{R}-10.9^{\circ}$, "44") and have quite similar lattice neighbor distances (5.6 $\AA \times 6 \AA$ and $6.1 \AA \times 5.9 \AA$, respectively) making them, in practice, hardly distinguishable. Typical images of the " 44 " structure are presented in Figure $4 \mathrm{a}$ and $4 \mathrm{~b}$ as along with the accepted model in Figure $4 \mathrm{c} .^{20}$ We note that as already known ${ }^{29}$ the appearance of a coincidence lattice is strongly dependent on both tunneling parameters and tip conditions (see Figures SI6 and SI7 in ESI).

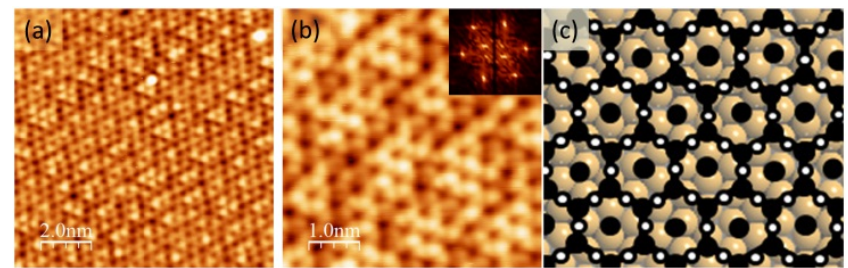

Figure 4. Topographic STM images of the " 44 ” or $\mathrm{Cu}_{2} \mathrm{O}(111)$-like structure at two magnifications (a) and (b). Inset in (b) is the FFT of the image. (c) O-centered honeycomb model describing the strained $\mathrm{Cu}_{2} \mathrm{O}(111)$-like structure with oxygen (black) and copper (white) over the substrate (golden). STM parameters: (a) I = $88 \mathrm{pA}$, Bias $=+1.4 \mathrm{~V}$, (b) I $=100 \mathrm{pA}$, Bias $=+0.97 \mathrm{~V}$.

To some extent, all the above described oxide structures have been already reported and, in particular, the " 44 " phase is still extensively studied because of its catalytic response. ${ }^{30}$ Interestingly, in spite of their different appearance and order range, the simplest model grouping them together consists of an hexagonal array of $\mathrm{Cu}$ and $\mathrm{O}$ atoms with periodicity of $6 \AA$. Depending on differences in local stoichiometry and defects, short or largely ordered oxide layers can be formed. Theoretical approaches based on densityfunctional theory (DFT) report the relative stability of different oxides surfaces on $\mathrm{Cu}(111)$ with varying $\mathrm{O}$ content that were considered as possible precursors of the bulk material. ${ }^{22,31}$ These precursors are viewed as ordered honeycomb arrays of $\mathrm{Cu}_{3} \mathrm{O}$ units 
with $\mathrm{O}$ adsorbed in nest positions. Despite the simplicity of the theoretical structures versus the complexity of the experimental landscape, the model offers understanding on the formation of the diverse oxide layers observed experimentally. On the one hand, the connected units form one trilayer (characteristic of bulk $\mathrm{Cu}_{2} \mathrm{O}$ ) that can be laterally adapted to give slightly strained or compressed coincidence lattice overlayers (see Figure SI7 in ESI). On the other hand, nucleation and growth at different locations accompanied by limited surface diffusion would importantly inhibit long-range order leading to a wide diversity of structures. As we will show next, the existence of such $\mathrm{Cu}_{3} \mathrm{O}$ units as building blocks of the oxide layers reported here is supported by the experimental findings.
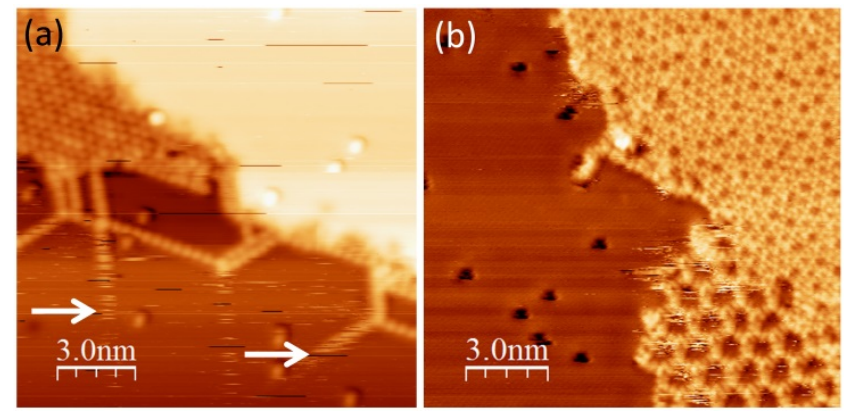

Figure 5. Topographic STM images of regions containing the " 44 ” and chain-like structures (a) and coexistence of short range honeycomb and a novel open honeycomb structure (b). STM parameters: (a) I = 200 pA, Bias $=-0.87 \mathrm{~V}$, (b) I = 62 pA, Bias $=+$ $0.50 \mathrm{~V}$.

Figure 5a shows some chain-like structures formed by rounded entities (small bright dots in the image) lying on the lower copper terrace as a prolongation of the "44" structure. They are straight nanostructures (nanowires) either free-ending or connected by one common unit. While the frizzy appearance of the free terminations (white arrows) is typical of movable species, meeting joints forming $120^{\circ}$ angles are consequence of the directional bonds expected between $\mathrm{Cu}_{3} \mathrm{O}$ units that provide rigidity to the assembly. Confined areas between chains evoke the fretwork rim seen in Figure 2f. Within the precursor's construction model, the defective short order honeycomb lattice may form by sequential incorporation of $\mathrm{Cu}_{3} \mathrm{O}$ units starting at different locations. In Figure 5b, coexisting with the short order honeycomb described in Figure 2, a considerably less dense and quite ordered structure is also observed (lower part of the image). As far as we know, this open honeycomb (OHC) structure has not been reported and constitutes the oxide layer with lower oxygen content encountered during the present investigation.
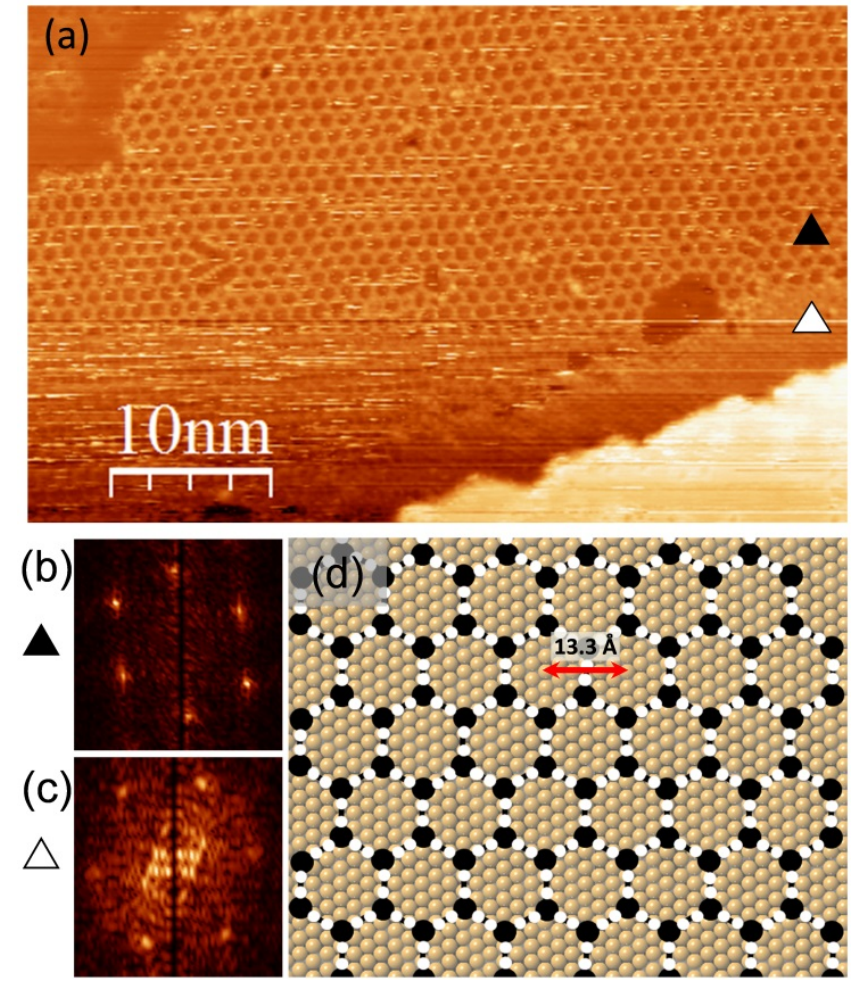

Figure 6. (a) STM topographic image showing a large region of the hexagonal open honeycomb, (b) and (c) FFTs of regions marked in (a). (d) Proposed model based on $\mathrm{Cu}_{3} \mathrm{O}$ units (c). STM parameters: $\mathrm{I}=110 \mathrm{pA}$, Bias $=-1.56 \mathrm{~V}$. 
The hexagonal OHC has a lattice parameter of $\approx 13.3 \AA$ and can be denoted in the Wood's notation as $(3 \sqrt{3} \times 3 \sqrt{3})$ R30 $0^{\circ}$ Though relatively defect-fee and large regions were observed this porous structure often contains different types of lattice defects (Figure 6a). As it can be seen from the Fast Fourier Transforms (FFT) taken on the two regions signaled in the image (Figures 6b and 6c) the OHC coexists with the "44" described in Figure 4. In fact, the new structure always exists side by side with other denser phases (see also Figures SI8 and SI9 in ESI). Therefore, we suggest for this new OHC a model coherent with the full understanding of copper oxidation as a sequential process in which every phase consists of a different arrangement of $\mathrm{Cu}_{3} \mathrm{O}$ units.

The structural model proposed for the OHC layer is depicted in Figure 6d, where white and black balls stand, respectively, for Cu and $\mathrm{O}$ atoms constituting the $\mathrm{Cu}_{3} \mathrm{O}$ units arranged in a honeycomb lattice on top of a $\mathrm{Cu}(111)$ substrate represented by golden balls. In the schematics, substrate and adlayer lattices are drawn at scale but atomic species are not. We note that accurate registry determination is not possible and atoms in the representation are placed on atop sites for simplicity.

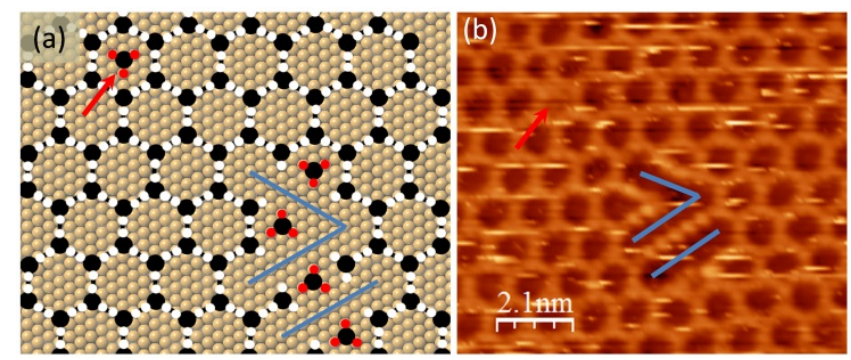

Figure 7. (a) Schematic representation of some topological defects (see text) observed in (b) magnified area of the STM image of OHC in Figure 6a. Red balls in (a) correspond to $\mathrm{Cu}$ atoms of displaced or interstitial $\mathrm{Cu}_{3} \mathrm{O}$.

The proposed model perfectly mimics the nearly defect-free layer and permits reproducing all topological defects observed. Typical defects as those observed in Figure 6 and magnified in Figure 7 are interpreted as missing or interstitial (red arrows) $\mathrm{Cu}_{3} \mathrm{O}$ units. Thus for instance groups of aligned vacancies (blue lines in Figures $7 \mathrm{a}$ and $7 \mathrm{~b}$ ) can be explained in terms of missing neighbor $\mathrm{Cu}_{3} \mathrm{O}$ entities. In fact, each couple of defects, missing plus interstitial units, are often found close together and might be understood by small displacements of complete units from the vacancy sites to nearby positions. It is worth mentioning that the linear structures seen at the central part of Figure 7b are similar to the chains observed in Figure 5a. Besides properties emerging from the particular oxide nature, the OHC nanostructure itself can be viewed as a two dimensional framework with well-defined pore size. This high quality open network can provide precise and confined localization of guest functional nano-objects (molecules, nanoparticles...). Compared to other bottom-up approaches, as supramolecular self-assembling in which open networks are made out molecules, the ability to generate two dimensional patterns without the need of additional organic building blocks and preserving the semiconductor/metal contact presents unquestionable advantages for device integration.

\section{Conclusions}

In the present work, by using an air-enriched bombardment method and interrupted oxidation, different oxide structures with distinct oxygen content have been obtained to partially cover the $\mathrm{Cu}(111)$ substrate. The coexistence of these oxide lattices on the same surface and the presence of bare copper terraces have allowed accurate identification of all reported oxide phases, their surface structure, local stoichiometry and oxygen content. Although the complex scenario arising from the oxidation of $\mathrm{Cu}(111) \mathrm{con}-$ firms the difficulties for fabricating a reproducible, stoichiometric metal/oxide interface, it can be understood as a whole. Starting from oxygen adsorption and after the first stages of oxide nucleation, oxidation seems to proceed passing from disordered and nonstoichiometric phases, until eventually the denser and highly ordered $\mathrm{Cu}_{2} \mathrm{O}(111)$-like lattice or “ 44 ” is formed.

Among other already described oxide structures, we report on a new phase consisting of an open honeycomb lattice which is viewed as a two dimensional framework with lattice parameter $\approx 13.3 \AA$ and the lower oxygen content reported so far. Based on existing theoretical models, we propose for this oxide layer a model consisting of indivisible $\mathrm{Cu}_{3} \mathrm{O}$ units. Similarly to the theoretical precursors, by oxygen incorporation the open network can evolve through different defective oxides to the most stable and compact bulk-like oxide surface.

Electronic Supplementary Information (ESI). Additional STM, LEED and XPS data for the clean Cu(111) and the oxide surface. Mass spectra (RGA) of the air-enriched Ar gas employed. Complementary STM images detailing the oxygen adsorption on Cu(111), the short order honeycomb and 5-7 defects formation, scanning dependence of the " 44 ” structure, coincidence lattice description and coexistence of the different oxide structures.

\section{ACKNOWLEDGMENT}

This work has been supported by the Spanish Government under project MAT2013-47869-C4-1-P and the Generalitat de Catalunya 2014 SGR501. We acknowledge the specific agreement between ICMAB-CSIC and the Synchrotron Light Facility ALBA and the Spanish MINECO through the project MAT2015-68994-REDC and the "Severo Ochoa" Program for Centers of Excellence in R\&D (SEV-20150496). We thank G. Sauthier for XPS measurements and Ll. Gines for equipment UHV support. 


\section{REFERENCES}

(1) Dagata, J.; Schneir, J.; Harary, H.H.; Evans, C.J.; Postek, M.T.; Bennett, J. Modification of hydrogen-passivated silicon by a scanning tunneling microscope operating in air. Appl. Phys. Lett., 1990, 56, 2001-2003.

(2) Garcia, R.; Martinez, R.V.; Martinez, J. Nano-chemistry and scanning probe nanolithographies. Chem. Soc. Rev., 2006, 35, $29-38$.

(3) Greiner, M. T.; Helander, M. G.; Tang, W. M.; Wang, Z. B.; Qiu, J.; Lu, Z. H. Universal energy-level alignment of molecules on metal oxides. Nat. Mat., 2011, 11(11), 76-81.

(4) Mittiga, A.; Salza, E.; Sarto, F.; Tucci, M.; Vasanthi, R. Heterojunction solar cell with $2 \%$ efficiency based on a $\mathrm{Cu}_{2} \mathrm{O}$ substrate. App. Phys. Lett., 2006, 88,163502.

(5) Olsen, L. C.; Addis, F. W.; Miller, W. Experimental and theoretical studies of $\mathrm{Cu}_{2} \mathrm{O}$ solar cells. Solar Cells, 1982, 7(3), 247-279.

(6) Sears, W. M.; Fortin, E. Preparation and properties of $\mathrm{Cu}_{2} \mathrm{O} / \mathrm{Cu}$ photovoltaic cells. Solar Energy Mat., 1984, 10(1), 93-103.

(7) Stampfl, C. Surface processes and phase transitions from ab initio atomistic thermodynamics and statistical mechanics. Catalysis Today, 2005, 105, 17-35.

(8) Stampfl, C.; Ganduglia-Pirovano, M. V.; Reuter, K.; Schefller, M. Catalysis and corrosion: The theoretical surface-science context. Surf. Sci., 2002, 500(1-3), 368-394.

(9) Hara, M.; Kondo, T.; Komoda, M.; Ikeda, S.; Shinohara, K.; Tanaka, A. $\mathrm{Cu}_{2} \mathrm{O}$ as a photocatalyst for overall water splitting under visible light irradiation. Chem. Comm., 1998, 2,357-358.

(10) Sinha, B.; Goswami, T.; Paul, S.; Misra, A. The impact of surface structure and band gap on the optoelectronic properties of $\mathrm{Cu}_{2} \mathrm{O}$ nanoclusters of varying size and symmetry. RSC Adv., 2014, 4(10), 5092-5104.

(11) Wilson, S. S.; Bosco, J.P.; Tolstova, Y.; Scanlon, D.O.; Watson, G. W.; Atwater, H.A. Interface stoichiometry control to improve device voltage and modify band alignment in $\mathrm{ZnO} / \mathrm{Cu}_{2} \mathrm{O}$ heterojunction solar cells. Energy Environ. Sci., 2014, 7(11), 3606-3610.

(12) Yang, F.; Choi, Y.; Liu, P.; Hrbek, J.; Rodriguez, J. A. Autocatalytic Reduction of a $\mathrm{Cu}_{2} \mathrm{O} / \mathrm{Cu}(111)$ Surface by CO: STM, XPS, and DFT Studies. J. Phys. Chem. C, 2010, 114(40), 17042-17050.

(13) Yang, F.; Choi, Y.; Liu, P.; Stacchiola, D.; Hrbek, J.; Rodriguez, J. A. Identification of 5 -7 Defects in a Copper Oxide Surface. J. Am. Chem. Soc., 2011, 133, 11474-11477.

(14) Matsumoto, T.; Bennett, R. A.; Stone, P.; Yamada, T.; Domen, K.; Bowker, M. Scanning Tunneling Microscopy Studies of Oxygen Adsorption on $\mathrm{Cu}(111)$. Surf. Sci. 2001, 471, 225-245.

(15) Wiame, F.; Maurice, V. ; Marcus, P.. Initial stages of oxidation of Cu(111). Surf. Sci., 2007, 601(5), 1193-1204.

(16) Pérez- León, C.; Sürgers, C.; Löhneysen, H. V. Formation of copper oxide surface structures via pulse injection of air onto Cu(111) surfaces. Phys. Rev. B, 2012, 85(3),035434.

(17) Albrecht, T.R.; Grütter, P.; Horne, D.; Rugar, D. Frequency modulation detection using high-Q cantilevers for enhanced force microscope sensitivity. J. Appl. Phys., 1991, 69, 668.

(18) Soon, A.; Todorova, M.; Delley, B.; Stamp, C. Thermodynamic stability and structure of copper oxide surfaces: A first-principles investigation. Phys. Rev. B, 2007, 75(12), 1-9.

(19) Ho, J. H.; Vook, R.W. (111)Cu그 growth modes on (111)Cu surfaces. J. Crys. Growth, 1978, 44, 561-569.

(20) Jensen, F.; Besenbacher, F.; Lægsgaard, E.; Stensgaard, I. Oxidation of Cu(111): two new oxygen induced reconstructions. Surf. Sci., 1991, 259, L774-L780.

(21) Due to modest implantation of residual nitrogen ions produced during the air-enriched $\mathrm{Ar}^{+}$sputtering, a small amount of copper nitride was observed (see STM images in Figure SI10 of ESI). Being out of the scope of the process reported here, full description of the nitride structure and references are given in the ESI.

(22) Soon, A.; Soehnel, T.; Idriss, H. Plane-wave pseudopotential density functional theory periodic slab calculations of CO adsorption on $\mathrm{Cu}_{2} \mathrm{O}(111)$ surface. Surf. Sci., 2005, 579(2-3), 131-140.

(23) Besenbacher, F. Scanning tunnelling microscopy studies of metal surfaces. Rep. Prog. Phys., 1996, 59, 1737-1802.

(24) Maroutian, T.; Degen, S.; Becker, C.; Wandelt, K.; Berndt, R. Phys. Rev. B, 2003, 68, 155414.

(25) Soon, A.; Todorova, M.; Delley, B.; Stampfl, C. Surface oxides of the oxygen-copper system: Precursors to the bulk oxide phase? Surf. Sci., 2007, 601, 5809-5813.

(26) Morita, S.; Giessibl, F. J.; Wiesendanger, R. Editors. Nanoscience and Nanotechnology. Noncontact Atomic Force Microscopy. SpringerVerlag, Berlin Hiedelberg, 2009

(27) Hashimoto, A.; Suenaga, K.; Gloter, A.; Urita, K.; Iijima, S. Direct evidence for atomic defects in graphene layers. Nature, 2004, 430(7002), 870-873.

(28) Meyer, J.C.; Kisielowski, C.; Erni, R.; Rossell, M.D.; Crommie, M.F.; Zettl, A. Direct imaging of lattice atoms and topological defects in graphene membranes. Nano Letters, 2008, 8(11), 3582-3586.

(29) Mönig, H.; Todorovi, M.; Baykara, M. Z.; Schwendemann, T.C.; Rodrigo, L.; Altman, E. I.; Pérez, R.; Schwarz, U. D. Understanding Scanning Tunneling Microscopy Contrast Mechanisms on Metal Oxides: A Case Study. ACS Nano, 2013, 7 (11), 10233-10244.

(30) Eren, B.; Lichtenstein, L.; Wu, Ch. H.; Bluhm, H.; Somorjai, G. A.; Salmeron, M. Reaction of CO with Preadsorbed Oxygen on Low-Index Copper Surfaces: An Ambient Pressure X ray Photoelectron Spectroscopy and Scanning Tunneling Microscopy Study. J. Phys. Chem. C, 2015, $119,14669-14674$

(31) Soon, A.; Todorova, M.; Delley, B.; Stamp, C. Oxygen adsorption and stability of surface oxides on Cu(111): A first-principles investigation. Phys. Rev. B, 2006, 73(16), 1-12. 


\title{
Supplementary Information
}

\section{Coming across a novel copper oxide 2D framework during the oxidation of $\mathrm{Cu}(111)$}

\author{
Sonia Matencio, ${ }^{\dagger}$ Esther Barrena and Carmen Ocal* \\ Institut de Ciència de Materials de Barcelona (ICMAB-CSIC) \\ Campus de la UAB, 08193 Bellaterra, Spain. \\ * Corresponding author \\ Email: cocal@icmab.es \\ ${ }^{\dagger}$ Present address: Institute of Experimental and Applied Physics, University of Regensburg, 93053 Regensburg, \\ Germany
}

\section{Clean Cu(111): STM, LEED and XPS}

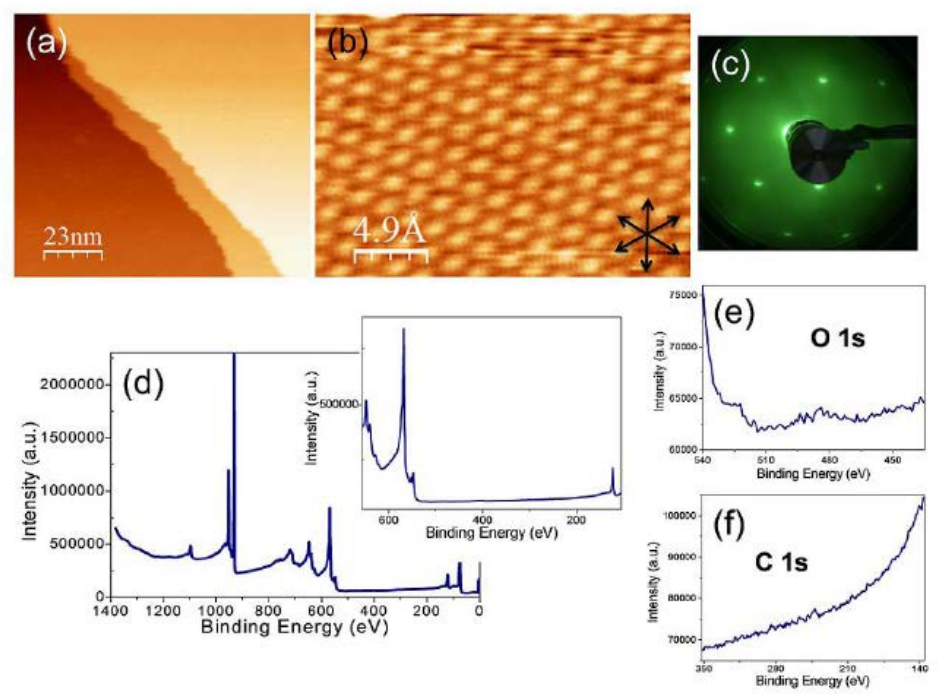

Figure SI1. Clean Cu(111) surface. Large (a) and small (b) topographic STM images: The monoatomic steps $\left(\mathrm{d}_{\mathrm{Cu}(111)}=2.1 \AA\right)$ in the large scale areas and the atomic resolution in small scans $\left(\mathrm{a}_{\mathrm{Cu}(111)}=2.56\right.$ $\AA$ ) were used for $\mathrm{XY}$ and $\mathrm{Z}$ scanner calibration to accurately determine the lattice parameters of the oxide structures. STM parameters: (a) I= 375 pA, Bias $=-1.40 \mathrm{~V}$ and (b) $\mathrm{I}=240 \mathrm{pA}$, Bias $=-0.30 \mathrm{~V}$. The arrows in (b) indicate the <111> directions of the $\mathrm{Cu}(111)$. (c) LEED pattern of the clean $\mathrm{Cu}(111)$. It served to check cleanness but also to univocally determine the relative orientation between the substrate and the different oxide lattices. After substrate cleaning, the XPS survey (d), O1s (e) and C1s (f) indicated no important traces of contaminants. 


\section{Air-enriched Ar gas}

Ambient air was introduced by means of a leak valve through the same pipe that the Ar gas, to the Ultra-High-Vacuum (UHV) preparation chamber. The pressure in the UHV chamber during oxidation was kept in the $10^{-5}$ mbar range and the gas mixture was ionized with the ion gun. The spectra corresponding to the residual gas analyzer (RGA) signal for a pressure of $10^{-6}$ mbar is shown in the figure. The peaks assignment is given in the figure caption.

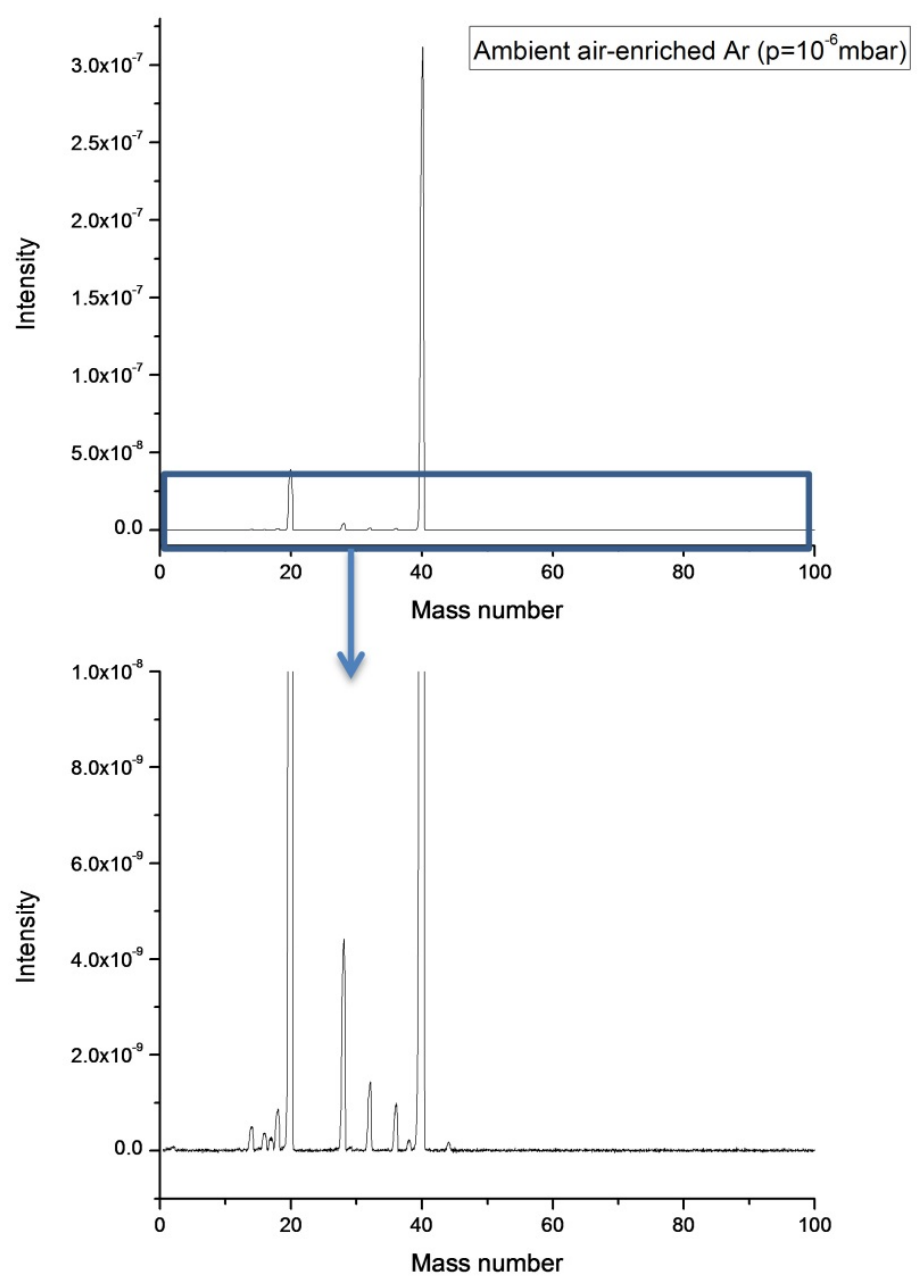

Figure SI2. Spectra of the residual gas in the preparation chamber during the entrance of ambient airenriched $\operatorname{Ar}\left(\mathrm{P}=10^{-6} \mathrm{mbar}\right)$. The main gas constituents are identified in the magnified spectrum as: $\mathrm{Ar}$ $(20,40), \mathrm{H}_{2} \mathrm{O}$ (group 16-18), $\mathrm{N}_{2}(28)$ and $\mathrm{O}_{2}(32)$ 


\section{Oxygen adsorption on $\mathrm{Cu}(111)$}

The adsorption of oxygen atoms on the $\mathrm{Cu}(111)$ has been observed both, after exposure to $\mathrm{O}_{2}$ at $\mathrm{RT}^{\mathrm{i}}$ and in the last stages of $\mathrm{Cu}_{2} \mathrm{O}(111)$ reduction by $\mathrm{CO}$. ${ }^{i i}$ Appearing either as isolated oxygen species or small oxygen clusters on the $\mathrm{Cu}(111)$ terraces, they are seen as depressions surrounded by concentric rings in the high resolution constant current STM images of Figure SI3, but as dark or bright spots depending on tip conditions. These rings correspond to oscillations (standing waves) in the electronic density of states of the cooper surface due to the oxygen adsorption (Friedel's oscillations). The adsorption site of the $\mathrm{O}$ atoms was determined to be threefold hollow ${ }^{i}$ from atomically resolved STM images. As it can be seen in Figure SI3b-c, these oxygen species can be moved by the sweep action of the tip during STM, indicating a weakly interaction with the unreconstructed substrate. This oxygen does not react at the copper terraces neither importantly attaches to the step edges. Thus, for instance the frizzy appearance of some steps is a clear indication of the metal atoms diffusion along oxygen free step edges. iiiviv The double height seen at some of the steps points to the beginning of oxidation described in the main text.

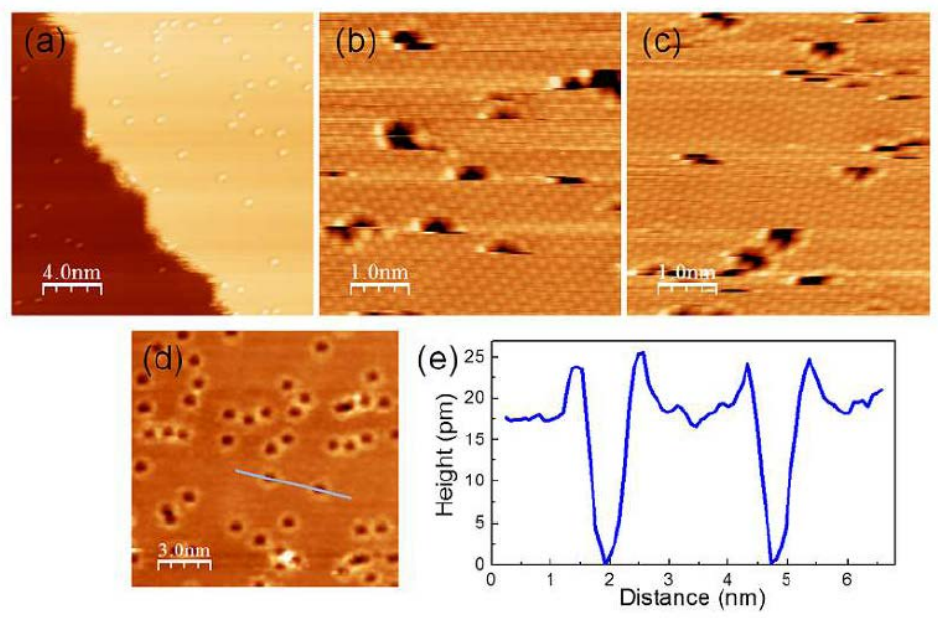

Figure SI3. Large scale topographic STM image of oxygen adsorbed in the Cu(111) surface (a). The high resolution images in (b) and (c) correspond to consecutive scans in the same area. A non-negligible drift during scan down (b) and up (c) is accompanied by a tip induced motion of the oxygen species. A medium scale image in (d) and the corresponding line profile (e) illustrate that these species are imaged as holes 15-20 pm deep and an apparent diameter of $~ 1 \mathrm{~nm}$. STM parameters: (a-d) I = 240 pA, Bias = $0.30 \mathrm{~V}$. 

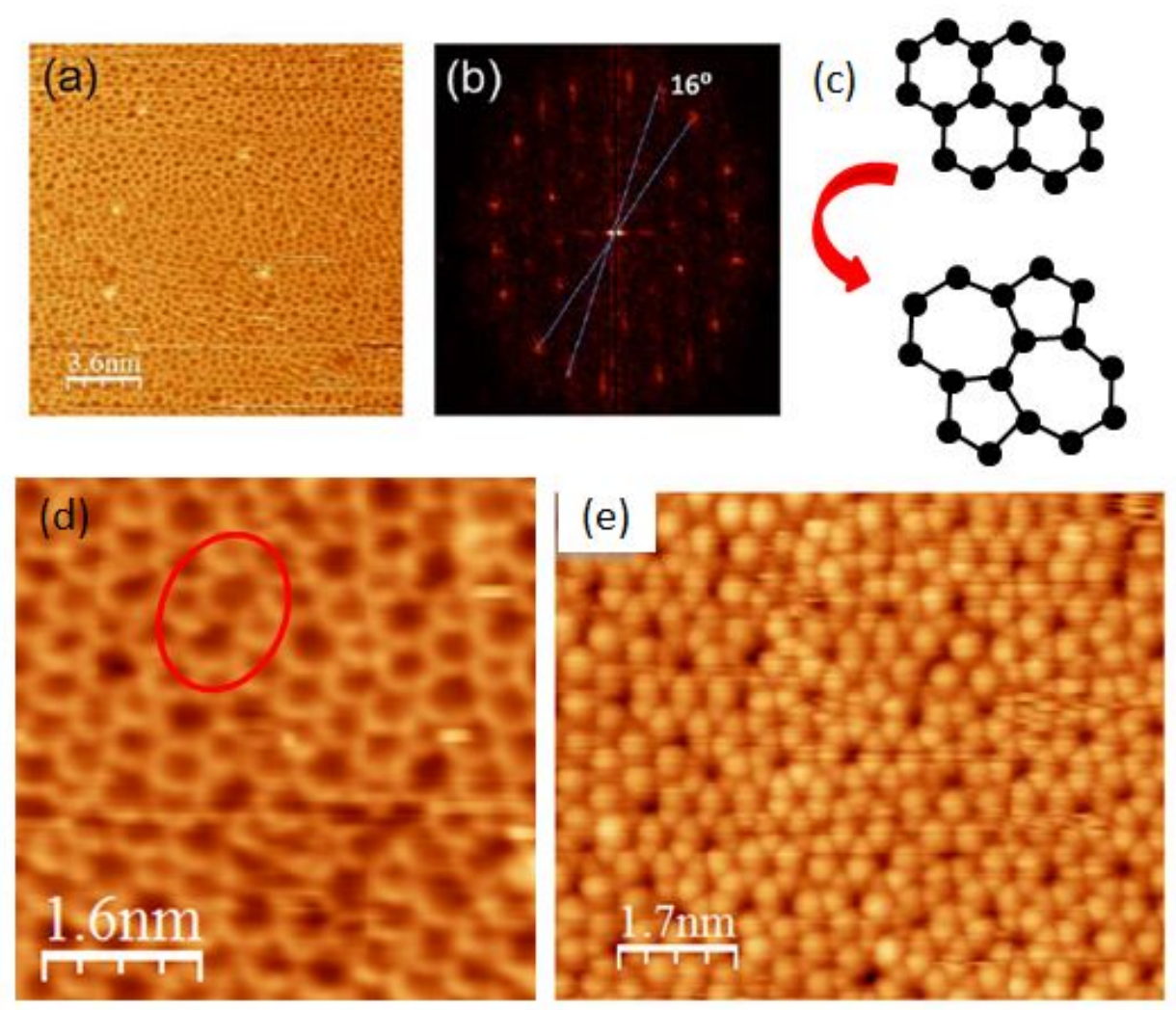

Figure SI4. (a, d, e) STM images of the defective or short order range honeycomb structure. Pentagons and heptagons are seen embedded in the honeycomb lattice (red circle in (d)). These topological 5-7 defects have been proposed to be analogous to the Stone-Wales (S-W) defects occurring, for instance, in graphene $^{v \text { vi }}$ and are generated by $90^{\circ}$ rotation of bonds about the midpoint. ${ }^{\text {vii }}$ Each oxide unit forming the lattice is depicted as a block dot in the schematic model of (c). Depending on the imaging resolution, the individual units could be seen (e). ${ }^{\text {viii }}$ STM parameters: (a, d) I = 194 pA, Bias $=-0.47 \mathrm{~V}$, (e) I = 80 $\mathrm{pA}$, Bias $=-0.53 \mathrm{~V}$.
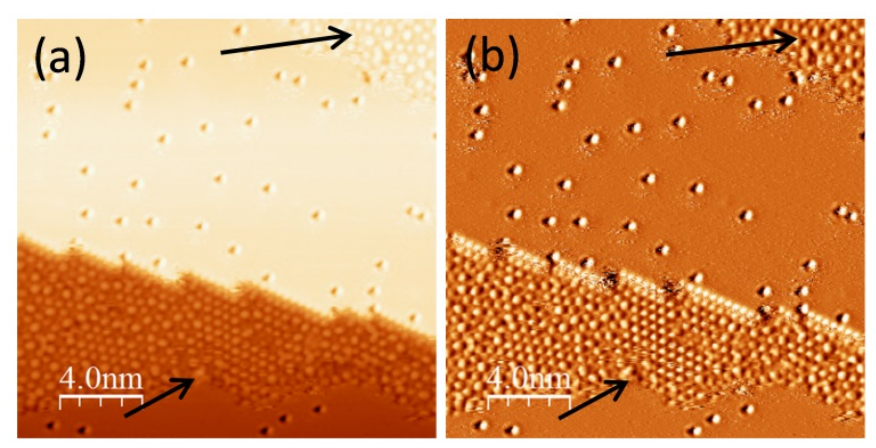

Figure SI5. STM topographic (a) and current (b) images of the O-deficient oxide structure at the edges of two consecutive substrate terraces. STM parameters: (a) I $=175 \mathrm{pA}$, Bias $=+1.70 \mathrm{~V}$. Note the nearly perfect hexagonal array formed by grouped neighboring oxygen vacancies. 


\section{Dependence of the “44” structure on imaging conditions}

Copper oxide STM images have been found to be strongly bias and tip conditions dependent. This is illustrated here by showing STM topographic images of the same " 44 " oxide structure at different tip and tunneling conditions. An impressive variation on the superlattice appearance is observed. Figure SI6a (Figure 4b in the main text) shows the honeycomb array result of a high resolution imaging with faint triangular groups of brighter/darker hexagons. The lattice is formed by bright dots in Figure SI6b which appear broadened forming a compact close packed lattice $(\sim 6 \AA)$ in Figures SI6c and 6d. These two later were found to transform the one into the other during scanning as illustrated in the bottom panels of the Figure. The arrows indicate the sense of fast scan direction. These changes can be only attributed to tip modifications during scanning (green to red arrows) and probably correspond to a tip with an adsorbate at the apex.

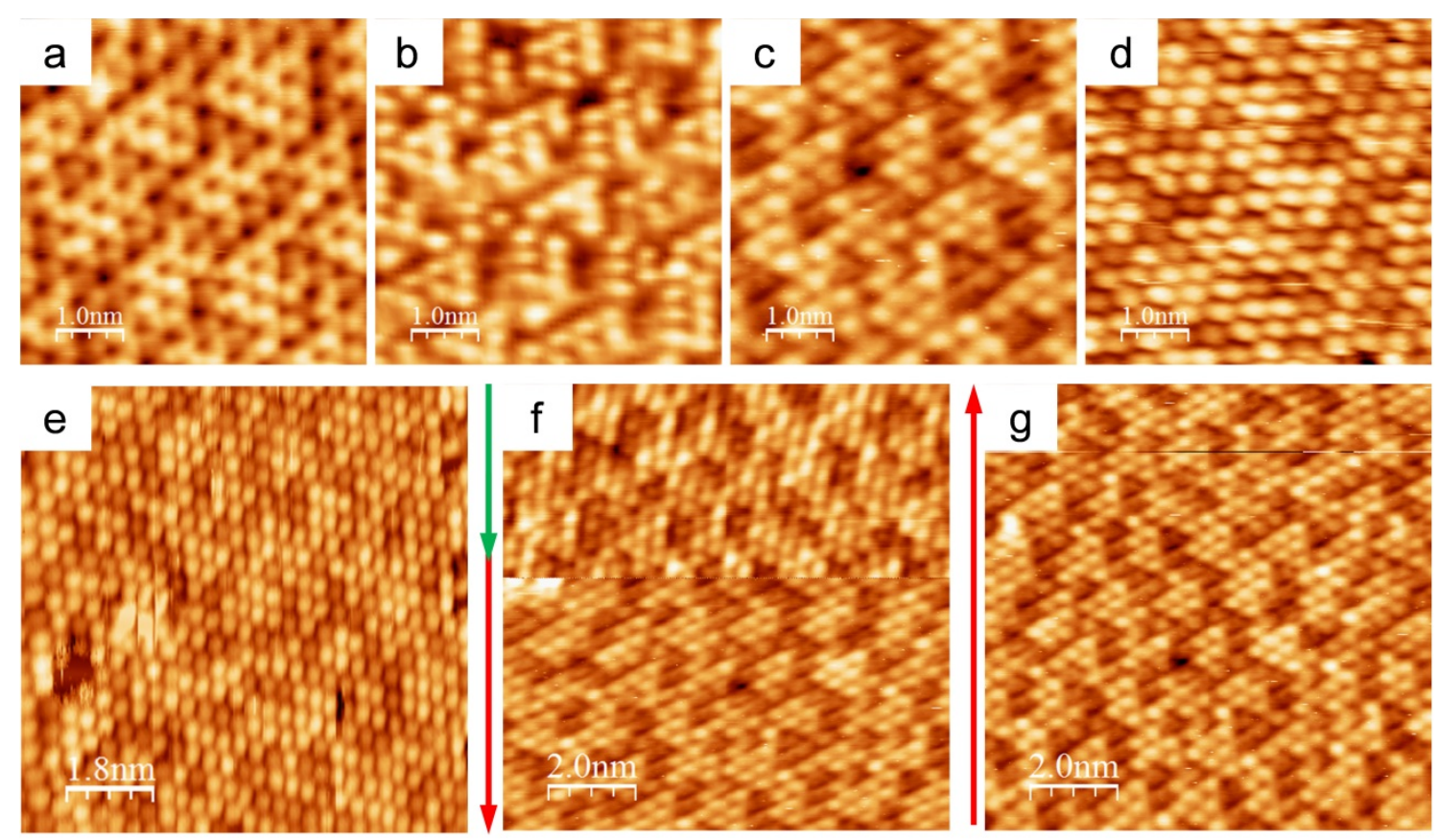

Figure SI6. Top: STM topographic images of the non-stoichiometric "44" structure obtained at three different tip conditions. (e)-(g) Consecutive STM images where the arrows indicate the fast scan direction. The arrow color change in (f) signals the point where resolution changes due to tip modification during scanning. STM parameters: (a) I $=100 \mathrm{pA}$, Bias $=+0.97 \mathrm{~V}$, (b) $\mathrm{I}=126 \mathrm{pA}$, Bias $=+1.20 \mathrm{~V}$, (c) $\mathrm{I}=338 \mathrm{pA}$, Bias $=-1.20 \mathrm{~V}$, (d) $-(\mathrm{e}) \mathrm{I}=$ $180 \mathrm{pA}$, Bias $=+0.97 \mathrm{~V}$, (f)-(g) $\mathrm{I}=340 \mathrm{pA}$, Bias $=+1.17 \mathrm{~V}$. 


\section{Coincidence lattice and Moiré patterns}

In addition to the bias and tip conditions dependence in STM and the easy occurrence of both, topological and stoichiometric defects, copper oxides imaging is quite troublesome because the large misfit between the oxide layer and the substrate leads, in practice, to similar but not identical surface structures and, commonly, site coincident lattice (SCL) or Moiré-like patterns can be observed. In these patterns, equivalent atoms of the overlayer adopt different sites on top of the substrate, therefore, exhibiting both a different local height reflected in true topographic differences and a different local density of stated (LDOS), this fact is reflected in a bias dependence. One of the proposed models to interpret such superstructures observed for the "44" is presented in Figure SI7a (drawing adapted from ref. ${ }^{\text {ix }}$ ) as a superlattice with a coincidence boundary at the $\mathrm{Cu}_{2} \mathrm{O}(111) / \mathrm{Cu}(111)$ interface. The oxide layer (oxide units represented by green dots) is strained to adopt the large misfit with the substrate. As three times of the overgrowth periodicity ( $3 \times 5.95 \AA=17.85 \AA$ ) nearly equals seven times the NN distance of the Cu substrate $(7 \times 2.56 \AA=17.92 \AA)$, the SCL described by the large oblique unit cell (dashed red line in the figure) is 9 times the oblique $\mathrm{Cu}_{2} \mathrm{O}$ unit cell (small rhomboid) and can be identified in Wood's notation by a $(7 \sqrt{3} \times 7 \sqrt{3}) \mathrm{R} 30^{\circ}$ or $\mathrm{c}(7 \times 7 \sqrt{3})$ superstructure of the $\mathrm{Cu}(111)$ (rectangular unit cell). Depending on the relative orientation between overlayer and substrate many different patterns may exist for the same overlayer structure (e.g., archetypical graphene Moiré patterns on (111) oriented metals). In the particular case of the copper oxide overlayers, the situation becomes even more complex. An experimentally observed $\mathrm{Cu}_{2} \mathrm{O}(111)$-like or "44" structure and the corresponding Fast Fourier Transform (FFT) are presented in Figure SI7b and SI7c, respectively. As it can be observed in the FFT, two concentric hexagonal lattices can be distinguished, the outermost pattern having a 7 times larger periodicity than the inner pattern. The separated respective FFTs and the inverse FFTs (IFFTs) are also shown (Figure SI6dg). The hexagonal lattice in Figures SI7d and SI7f corresponds to the characteristic $\sim 6 \AA$ distance of the $\mathrm{Cu}_{2} \mathrm{O}$, while the large lattice is result of the large superstructure of this example.
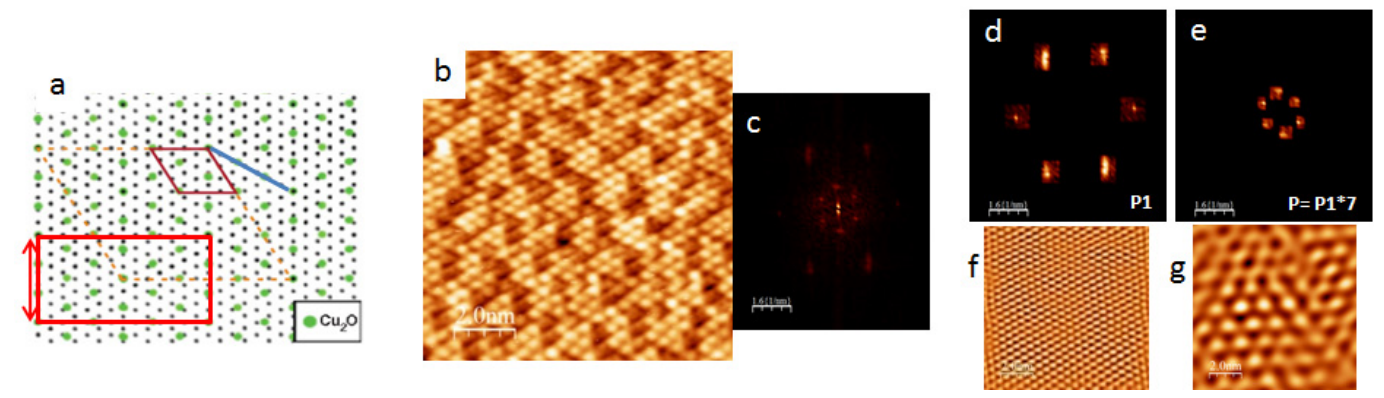

Figure SI7. (a) Model for a coincident site lattice of the $\mathrm{Cu}_{2} \mathrm{O}(111)$-“44” structure on $\mathrm{Cu}(111)$. ${ }^{\mathrm{x} x \mathrm{i}}$ For simplicity, green dots stand for oxide units forming the oxide layer and black small dots represent the underlying substrate (drawing adapted from ref. 9). Depicted unit cells are described in the text. (b) Topographic STM image of the non-stoichiometric “44" structure and (c) corresponding FFT. (d) and (e) are the separated hexagonal lattices of (c) and (f) and (g) the corresponding inverse FFTs. STM parameters: (b) $\mathrm{I}=340 \mathrm{pA}$, Bias $=+1.17 \mathrm{~V}$. 


\section{Coexistence of oxide layers with different oxygen content}

As commented in the main manuscript, all observed structures coexist on one or other regions.
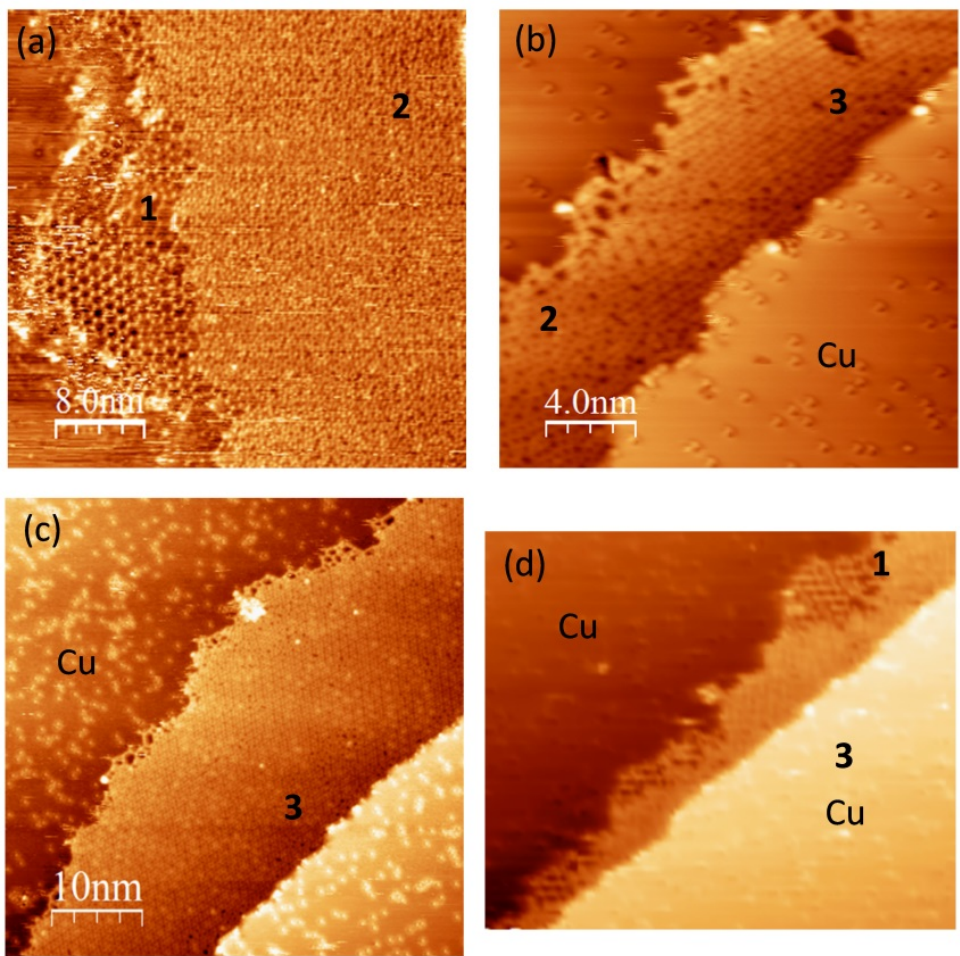

Figure SI8. STM images of the partially oxidized $\mathrm{Cu}(111)$ showing that the different structures described in the main text coexist on the surface. Increasing numbers labeling the structures indicate increasing oxygen content: (1) open honeycomb (OHC), (2) short order honeycomb (SOHC) and (3) compact "44" or $\mathrm{Cu}_{2} \mathrm{O}(111)$-like. STM parameters: (a) $=94$ pA, Bias $=-0.90 \mathrm{~V}$, (b) $\mathrm{I}=187 \mathrm{pA}$, Bias = $-1.40 \mathrm{~V}$, (c) $\mathrm{I}=62 \mathrm{pA}$, Bias $=-1.40 \mathrm{~V}$, (d) $\mathrm{I}=117 \mathrm{pA}$, Bias $=-1.40 \mathrm{~V}$.
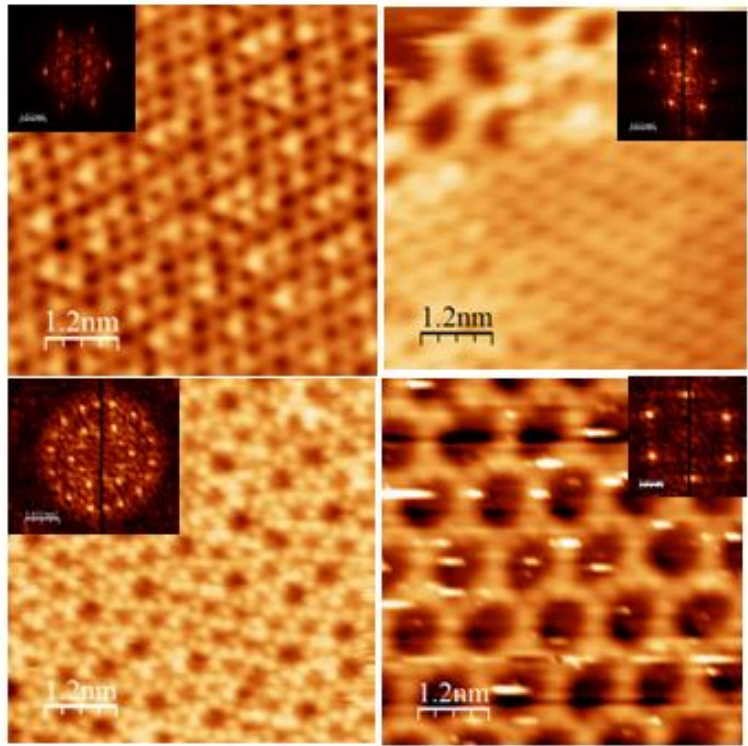

Figure SI9. High resolution STM images of the different oxide structures and their corresponding Fast Fourier Transforms (FFT). 


\section{Residual incorporation of nitrogen}

It has been reported that nitrogen does not chemisorbed on copper directly unless this process is activated by ion implantation or atomic beam sources. Though the ionization energy for nitrogen is higher than for oxygen, nitrogen ionized species would exist during the air-enriched procedure employed in the present investigation.

Previous studies on the incorporation of nitrogen in $\mathrm{Cu}(111)$ indicate that the ordered structure experimentally observed is a pseudo-(100) surface phase with a rectangular unit cell. ${ }^{11-15}$ As demonstrated by DFT calculations, ${ }^{16}$ this structure is highly energetically favorable. Though scarcely, we observed small surface regions (Figure SI10) presenting a surface structure in agreement with copper nitride formation. This $\mathrm{CuN}$ layer has been interpreted by insertion nitrogen atoms between $\mathrm{Cu}$ atoms in the surface. Because the insulating character of the nitride layer, in the constant current STM images of Figure SI10, it appears lower than the level of the original $\mathrm{Cu}$ terrace (see profile in d). The difference in height depending on the measuring parameters. ${ }^{15}$ The nitride lattice has a $(25 \times 7 \sqrt{3})$ rect unit cell well known, clearly identifiable and distinguishable from all the hexagonal and honeycomb structures developed during copper oxide formation.
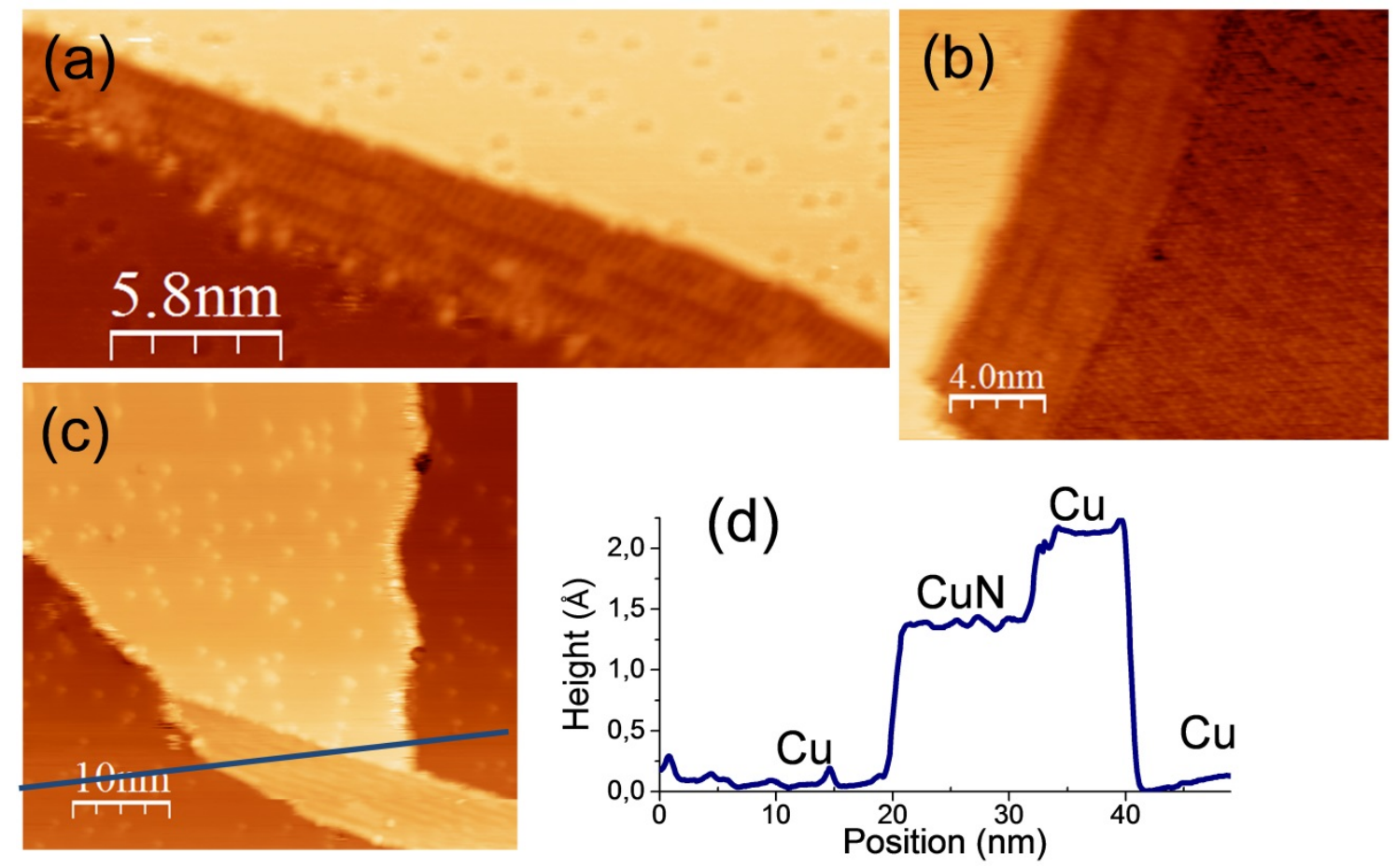

Figure SI10. STM images of a region of a $\mathrm{Cu}(111)$ where residual incorporation of nitrogen is observed. STM parameters: $I=200 \mathrm{pA}$, Bias $=-0.80 \mathrm{~V}$. The images reveal the $(25 \times 7 \sqrt{3})$ rect. coincidence surface mesh resulting from pseudo-(100) layer and the underlying (111) substrate. The modulation or regular wavy bands running along close-packed $<110>$ directions of the substrate characteristic of the coincidence mesh are accompanied for a small angular distortion $\left(\phi \approx 14^{\circ}\right.$ in our case) of the CuN super cell away from the $<110>$ direction of $\mathrm{Cu}$ substrate results in a long-range modulation disorder. ${ }^{15}$ 


\section{REFERENCES}

(') F. Wiame, V. Maurice, P. Marcus. Initial stages of oxidation of $\mathrm{Cu}(111)$. Surface Science, 601(5), 1193-1204, 2007

(ii) F. Yang, Y. Choi, P. Liu, J. Hrbek, J. A. Rodriguez. Autocatalytic Reduction of a $\mathrm{Cu}_{2} \mathrm{O} / \mathrm{Cu}(111)$ Surface by CO: STM, XPS, and DFT Studies. The Journal of Physical Chemistry C, 114(40), 17042-17050, 2010

(iii) M. Giesen-Seibert, R. Jentjens, M. Poensgen, and H. Ibach. Time dependence of step fluctuations on vicinal Cu(1119) surfaces investigated by tunneling microscopy. Physical Review Letters, 71(21):3521-3525, 1993

( ${ }^{\text {iv }) ~ M . ~ G i e s e n-S e i b e r t, ~ F . ~ S c h m i t z, ~ R . ~ J e n t j e n s, ~ a n d ~ H . ~ I b a c h . ~ T i m e ~ f l u c t u a t i o n s ~ o f ~ s t e p s ~ o n ~} \mathrm{Cu}(11 \mathrm{n})$ surfaces investigated by temperature variable tunneling microscopy. Surface Science, 329(1-2):47-60, 1995

( $v$ ) A. Hashimoto, K. Suenaga, Al. Gloter, K. Urita, and S. Iijima. Direct evidence for atomic defects in graphene layers. Nature, 430(7002):870-873,2004.

( $\left.{ }^{\mathrm{vi}}\right)$ J. C. Meyer, C. Kisielowski, R. Erni, M. D. Rossell, M. F. Crommie, and A. Zettl. Direct imaging of lattice atoms and topological defects in graphene membranes. Nano Letters, 8(11):3582-3586, 2008.

( ${ }^{\text {vii }) ~ G . ~ R a j a s e k a r a n ~ a n d ~ A v i n a s h ~ P a r a s h a r . ~ M o l e c u l a r ~ d y n a m i c s ~ s t u d y ~ o n ~ t h e ~ m e c h a n i c a l ~ r e s p o n s e ~ a n d ~ f a i l u r e ~}$ behaviour of graphene: performance enhancement via 5-7-7-5 defects. RSC Adv., 2016, 6, 26361-26373.

(viii) F. Yang, Y. Choi, P. Liu, D. Stacchiola, J. Hrbek, J. A. Rodriguez. Identification of 5 -7 Defects in a Copper Oxide Surface. Journal of the American Chemical Society, 133, 11474-11477, 2011.

$\left({ }^{i x}\right)$ C. Pérez- León, C. Sürgers, H. V. Löhneysen. Formation of copper oxide surface structures via pulse injection of air onto Cu(111) surfaces. Phys. Rev. B, 85(3),035434, 2012.

$\left.{ }^{\mathrm{x}}\right)$ J. H. Ho and R. W. Vook, (111) $\mathrm{Cu}_{2} \mathrm{O}$ growth modes on (111)Cu surfaces J. Crystal Growth 44, 561 (1978).

( $\left.{ }^{x i}\right)$ F. Jensen, F. Besenbacher, E. Lægsgaard, and I. Stensgaard, Oxidation of Cu(111): two new oxygen induced reconstructions. Surf. Sci. 259, L774 (1991).

(12) S.M. Driver, Ph Woodruff. Nitrogen-induced pseudo-(100) reconstruction of the $\mathrm{Cu}(111)$ surface identified by STM. Surf. Sci. 442, 1-8 (1999)

(13) J.F. Skelly, T. Bertrams, A.W. Munz, M.J. Murphy, A. Hodgson. Nitrogen induced restructuring of $\mathrm{Cu}(111)$ and explosive desorption of $\mathrm{N}_{2}$. Surf. Sci. 415, 48-61 (1998)

(14) A.M. Goedken, S.L. Silva, S.M. York, F.M. Leibsle STM studies of the N/Cu(111) system: surface structure,electron confinement and tip-induced modification. Eur. Phys. J. AP 19, 77-82 (2002)

(15) H. Baek, S. Jeon, J. Seo, Y. Kuk. Geometric and Electronic Structure of Passive CuN Monolayer on $\mathrm{Cu}(111)$ : A Scanning Tunneling Microscopy and Spectroscopy Study. J. Korean Phys. Soc. 56 ( 2), 620624 (2010)

(16) A. Soon, L. Wong, M. Lee, M. Todorova, B. Delley, C. Stampfl. Nitrogen adsorption and thin surface nitrides on $\mathrm{Cu}(111)$ from first-principles Surf. Sci. 601, 4775-4785(2007) 